\title{
Die Gemeinsame Landesplanung (GL) von Berlin und Brandenburg - eine tragfähige Institution für die Region?
}

\author{
Markus Vette
}

\section{Aufgabe}

Häufig wird für die Zukunft des Landes Brandenburg die beabsichtigte Länderfusion mit Berlin als strategische Zielstellung genannt. Bei einer solchen möglichen Länderfusion werden zunächst formal eine Regierung, ein Parlament und eine Landesverwaltung entbehrlich bzw. entsprechend neue Strukturen für ein gemeinsames Land müssen entstehen. Forderungen nach Personaleinsparungen in der Verwaltung von Brandenburg und Berlin in erheblichem Umfang sollen aber nicht den Blick davor verstellen, dass gleichzeitig qualitative Anforderungen an die Mitarbeiter der Verwaltung einer zukunftsfähigen Hauptstadtregion wachsen! Der vorliegende Artikel befasst sich damit, welche Chancen und Risiken in einer solchen Fusionsabsicht liegen. Nach historischen Bezuigen und Beispielen wird gefragt, die gegenwärtige Situation wird analysiert, Vergleiche aus anderen Regionen in Deutschland werden kritisch herangezogen, die beabsichtigte Senderfusion von ORB und SFB wird skizziert. Insbesondere wird die Struktur der Gemeinsamen Landesplanung $(\mathrm{GL})$ beider Bundesländer, die in der Bundesrepublik Deutschland ohne Beispiel ist und vielleicht gerade deshalb beispielgebend sein kann, erörtert.

\section{Fusionen als Lösungskonzept für Regionen?}

Gegenwärtig kann man in vielen Bereichen der Gesellschaft die Suche nach neuen Strukturen beobachten, die im Zuge der Globalisierung bzw. des europäischen Einigungsprozesses gesucht und auch gefunden werden: Daimler fusioniert mit Chrysler, Vodafone mit Mannesmann, die Gewerkschaft ver.di entstand, aus dem Süddeutschen Rundfunk und dem Südwestfunk entstand der Südwestrundfunk. Man will sich „neu aufstellen“, neue Potenziale erschließen, neue Märkte strukturieren. Freilich entstehen in diesem Zusammenhang Fragen: Sind bei Daimler und bei Chrysler nur Gewinner anzutreffen? Weshalb wird der im Südwesten der Bundesrepublik gelegene kleine Saarländische Rundfunk ${ }^{1}$ „nicht gleich mitfusioniert"?

Sollen also Berlin und Brandenburg fusionieren, unter welchen Bedingungen? Hier soll der Frage nachgegangen werden, ob es eine adäquate institutionell verankerte Möglichkeit des gemeinsamen Handelns von Berlin und Brandenburg gibt, speziell mit oder in der GL beider Bundesländer.

\section{Geschichtliches zum Verhältnis von Berlin und Brandenburg}

Berlin und Brandenburg verdienen in vielerlei Hinsicht eine differenzierte Betrachtung. Die Metropole mit etwa 3,5 Mio. Einwohnern dominiert klar gegenüber 2,5 Mio. Einwohnern im Land Brandenburg. Nahe Berlin, „im Speckgürtel“, sind Zuwachs und Zuzug zu verzeichnen, in den äußeren Regionen des Landes Brandenburg ziehen vor allem die jungen Menschen weg, wie das Statistische Jahrbuch belegt. Von der Sozialisation her gibt es eine östlich geprägte Mehrheit, die einer Mentalität des alten Westteils der Stadt gegenübersteht. Auf der politischen Ebene ist eine Dominanz von Vertretern des westlichen Teils gegenuiber einer schwachen Organisationsstruktur in den östlichen Stadtteilen festzustellen. Dass für Fusionsabsichten gerade somit mehrfache Konfliktlinien bestehen, liegt auf der Hand.

Der Status von Berlin in der Provinz Brandenburg des Staates Preußen war bereits historisch ein besonderer. Wenn man mit der Neukonstituierung des preußischen Staates 1815 beginnt, so bestand Berlin als eigener Regierungsbezirk nur von 1816 bis 1821 . Danach gingen Leitungsfunktionen an den Oberpräsidenten (von Berlin und der Provinz Brandenburg) in Potsdam über. Nach der Reichsgründung 1871 schied Berlin 1875 aus dem Provinzialverband Brandenburg aus, doch gelang die Bildung einer eigenen Provinz Berlin nicht. Zur Jahrhundertwende expandierte Berlin. Das damalige Berlin konnte zum 1. April $1912^{2}$ einen Zweckverband GroßBerlin mit dem nördlichen Landkreis Niederbarnim und dem südlichen Landkreis Teltow schaffen, seine Aufgaben waren die Regelung des öffentlichen Schienenverkehrs, die Bauplanung, Erwerb und Erhalt von Freiflächen wie Wald und Parkanlagen, was gesetzlich geregelt war. Erst nach dem Ersten Weltkrieg konnte am 27. April 1920 das Gesetz über die Bildung einer neuen Stadtgemeinde Berlin beschlossen werden. Der Name „Groß-Berlin“ wurde 1920 vermieden und wurde erst nach 1945 , dann für die östliche Hälfte, die spätere Hauptstadt der DDR, verwendet. Mit dem Mauerbau von 1961 wurden die Verbindungen des Westteils der Stadt zum Ostteil sowie zum Umland abgeschnitten. Aus der Zeit der Teilung stammen viele sowohl in Westberlin als auch in Ostberlin entstandene Institutionen und Strukturen, die mit der deutschen Einheit 1990 nicht nur miteinander konkurrierten, sondern gegenüber dem strukturschwachen umgebenden Brandenburg in der Regel dominierten, so etwa im Bereich der Wissenschaft und Kultur. In Berlin wurde ein umfangreicher Prozess der Umstrukturierung der Verwaltung in Angriff genom- 
men, so eine Reihe von Schritten zur Verwaltungsreform. Dafür wurden mehrere gesetzliche Regelungen geschaffen, etwa das Verwaltungsreformgrundsätzegesetz.

\section{Rechtsgrundlagen für die Gemeinsame Landesplanung}

Die Rechtsgrundlagen für die gemeinsame Landesplanung von Berlin und Brandenburg reichen bis ins Jahr 1995 zurück: Im Herbst 1994 wurde die Fusion zwischen Berlin und Brandenburg zu einem der strategisch entscheidenden Projekte der Brandenburger Landesregierung erklärt, die von einer absoluten Mehrheit der SPD getragen wurde. Die Fusionsabsicht wurde in der ersten Regierungserklärung des Brandenburger Ministerpräsidenten Mitte November 1994 artikuliert, in der im Dezember 1994 erfolgten Aussprache ${ }^{3}$ zu dieser Regierungserklärung spielte dieses Thema jedoch keine dominierende Rolle. Man könnte meinen, dass einigen Parlamentariern die Tragweite dieser Fusionsabsicht verschlossen blieb, zumal Sondierungen und Verhandlungen Sache der Brandenburger Landesregierung, also gerade nicht des Parlamentes waren. Im Brandenburger Parlament wurde ein Unterausschuss ${ }^{4}$ Berlin-Brandenburg (des Hauptausschusses) gebildet, darüber hinaus kamen parlamentarische Instrumente der Kontrolle, so die Kleine und Mündliche Anfrage, zum Stand und Fortgang der Verhandlungen zur Anwendung. Die Einsetzung einer Enquetekommission ${ }^{5}$ erfolgte nicht. Die in Brandenburg zu diesem Zeitpunkt gleich starken beiden Oppositionsfraktionen von CDU und PDS verhielten sich unterschiedlich: Die CDU befürwortete weitestgehend die beabsichtigte Länderfusion, ohne auf deren Erarbeitung einen merklichen Einfluss nehmen zu können, die oppositionelle PDS lehnte die Länderfusion ab, mit Verweis auf den dann ausgearbeiteten Neugliederungsstaatsvertrag.

1995 wurde der Landesplanungsvertrag ${ }^{6}$ abgeschlossen . Auf der Grundlage dieses Staatsvertrages gibt es Rechtsverordnungen, so eine gemeinsame Raumordnungsverfahrensverordnung. Von 1995 stammt auch der Landesentwicklungsplan Brandenburg Zentralörtliche Gliederung7 $^{7}$ (LEP I) - er ist noch kein gemeinsamer Plan. Das Gemeinsame Landesentwicklungsprogramm der Länder Berlin und Brandenburg8 (LEPro) stammt von 1997. Aus 1998 stammt der Gemeinsame Landesentwicklungsplan für den engeren Verflechtungsraum Brandenburg-Berlin ${ }^{9}$ (LEP eV). Für den geplanten Großflughafen Berlin-Brandenburg International wurde ein Gemeinsamer Landesentwicklungsplan Standortsicherung Flughafen ${ }^{10}$ (LEP SF) erarbeitet. Nicht vergessen werden darf das Brandenburger Gesetz zur Regionalplanung und zur Braunkohlen- und Sanierungsplanung ${ }^{11}$ (RegBkPIG) zuletzt geändert im März 2001.

Organisation und Aufbau der Gemeinsamen Landesplanung wurden durch eine Verwaltungsvereinbarung ausgestaltet. Insbesondere wurde auf eine von beiden Partnern angestrebte Zusammenarbeit an der Spitze der Abteilung und in ihren Referaten geachtet, indem der
Leiter von Brandenburg und sein ständiger Stellvertreter von Berlin ernannt wurden. Diese „symmetrische“ Strukturplanung zu einem einvernehmlichen Handeln soll jedoch nicht darüber hinwegtäuschen, dass unterschiedliche Voraussetzungen in beiden Bundesländern zu beobachten waren: So konnte Berlin, wo das Stadtstaatenprivileg den Raumordnungsplan durch einen Flächennutzungsplan ersetzt, bereits einen solchen Flächennutzungsplan in Kraft setzen und in diesem Sinne wichtige eigene Belange vorab selbst regeln. Dagegen waren im Land Brandenburg zahlreiche Planungen, so fuir einen internationalen Flughafen, die kommunale Bauleitplanung, die Regionalplanung und nicht zuletzt die Landesplanung sowie anderes mehr - man denke an die Konversion - noch im Gange, es konnten also zum Zeitpunkt des Vertragsabschlusses noch nicht Entscheidungen aus eigener Kraft erfolgen und in Kraft gesetzt werden. So überrascht es nicht, dass die parlamentarische Kontrolle des Regierungshandelns im Falle der Raumordnung nach den Erfahrungen der Mitarbeiterinnen und Mitarbeiter der Gemeinsamen Landesplanung häufiger durch die Brandenburger Parlamentarier erfolgte: Dort waren die Probleme eben noch zu lösen. Die Gemeinsame Landesplanung unterliegt auch der Kontrolle durch den Landesrechnungshof, in diesem Falle wird durch eine Verwaltungsvereinbarung beider Landesrechnungshöfe ein Landesrechnungshof im Benehmen mit dem anderen tätig.

Für Berlin und Brandenburg wurde gerade kein Planungsverband geschaffen. - Dagegen gibt es eine Landesplanungskonferenz, paritätisch zusammengesetzt je aus Regierungschef, Chef der Staatskanzlei, Innenminister, Verkehrs- und Bauminister, Landwirtschaftsminister und Wirtschaftsminister. Da die Strukturen der Ministerien und Senatsverwaltungen nicht äquivalent sind, ist auf Berliner Seite auch der Justizsenator Mitglied dieser Landesplanungskonferenz. Wie arbeitet die Gemeinsame Landesplanung aber im Konfliktfall? Im Falle, dass die beiden Bundesländer unterschiedliche Positionen vertreten, wenden sich der Leiter der GL und sein „ständiger Vertreter“ 12 an beide Staatssekretäre, gegebenenfalls anschließend an den Minister und den Senator. Schließlich bleibt noch die benannte Landesplanungskonferenz im Konfliktfall anzurufen, um ein länderübergreifendes Einvernehmen zu erzielen. Aus der Praxis der Arbeit der Gemeinsamen Landesplanung kann berichtet werden, dass dieser Weg der Konfliktlösung bislang einmal bis zur Ebene des Senators bzw. Ministers beschritten werden musste, und zwar bei dem Abschluss des Raumordnungsverfahrens für das Transrapid-Projekt.

\section{Planungen der anderen Regionen mit Metropolen in Deutschland}

Besonders lange und umfangreiche Planungen von Städten in einer gemeinsamen Region findet man im Ruhrgebiet. Die Nähe der Städte war Anlass für die gemeinsame Planung, es gibt eine umfangreiche Planungsgeschichte. Bekannt ist der Siedlungsverband Ruhr (SVR), 1920 vom preußischen Staat gegründet, der über die 
Ansiedlung von 150000 Kumpeln ${ }^{13}$ zur Realisierung der Kohlelieferungen an den Kriegssieger Frankreich die Region prägte und der über die Jahrzehnte eine immer größere Wirksamkeit erreichte und damit durchaus in eine Konkurrenzposition gegenüber der Regierung gelangte. Nicht einmal die Nazis wagten die Auflösung dieses Verbandes, sie veränderten lediglich die Mehrheitsverhältnisse zu ihren Gunsten. 1975 wurden Bezirksplanungsräte gebildet, 1979 wurde der Kommunalverband Ruhrgebiet mit geringeren Kompetenzen als Nachfolger des SVR eingerichtet.

Im Großraum Frankfurt am Main gab es 2001 Bestrebungen zur Vergrößerung des Planungsverbandes: Dem Umlandverband Frankfurt UVF folgte als Rechtsnachfolger der Planungsverband Frankfurt Region Rhein-Main. Er erstellt einen regionalen Flächennutzungsplan, kombiniert also die Funktion des Flächennutzungsplanes mit der des Regionalplanes. Dies ist im Hessischen Landesplanungsgesetz vom Dezember 2000 geregelt.

In Hannover wurde eine „Region Hannover“ mit Kompetenzen u. a. für Verkehr, Abfall, Freiraumplanung und regionale Planung gebildet.

Auch in München ist eine regionale Planungsgesellschaft gegründet worden: Der Regionale Planungsverband München ist ein gesetzlich vorgeschriebener Pflichtverband mit 186 Städten und Gemeinden und acht Landkreisen. Die Landeshauptstadt München kann mit $40 \%$ Stimmenanteil nicht die Partner, also die Städte oder die Landkreise, majorisieren. Zusätzlich gibt es mit dem Planungsverband „Äußerer Wirtschaftsraum München“ einen freiwilligen Zweckverband, die gegenüber dem Regionalen Planungsverband etwas unterschiedlichen Stimmenanteile gestatten aber auch hier keine Majorisierung durch die Metropole.

Auffallend sind bei diesen Bestrebungen anderer deutscher Metropolen zur Neustrukturierung des Verhältnisses zu ihrem „Umland“, dass man sich als Region positionieren will, auch im europäischen Kontext. Ob im Falle einer erfolgversprechenden deutschen Olympiabewerbung für 2012 ein weitergehender Planungsschub erforderlich ist, wird zu beobachten sein. Diese genannten Metropolen ${ }^{14}$ in anderen deutschen Regionen dominieren jedoch in ihrem Verhältnis zum Umland nicht so deutlich wie dies bei Berlin gegenüber Brandenburg der Fall ist. Insofern ist die Kenntnis dieser regionalen Planungen wichtig, eine formale unreflektierte Übernahme dieser Planungsverbände wird jedoch der Situation in Berlin und Brandenburg nicht gerecht.

\section{Senderfusion statt Länderfusion in Berlin und Brandenburg?}

Durch die politischen Parteien in Berlin und Brandenburg wird etwa seit dem Jahr 2000 die Fusion beider Landesrundfunkanstalten, des SFB und des ORB, betrieben. Ist die Fusion dieser öffentlich-rechtlichen Rundfunkanstalten ein Beispiel oder Vorbild für die Fusion der Länder? Zunächst muss beachtet werden, dass der öffentlichrechtliche Rundfunk über Gebühren (sowie Werbeeinnahmen) finanziert wird, sodass diese beabsichtigte
Senderfusion keine finanziellen Lasten für die ohnehin strapazierten öffentlichen Haushalte beider Bundesländer darstellt. Mit einem summarischen Anteil von etwa sieben Prozent am ARD-Gemeinschaftsprogramm kann die Region in Berlin und Brandenburg durchaus deutlicher identifiziert werden als gegenwärtig. Man würde etwa die Stärke des Hessischen Rundfunks innerhalb des ARD-Verbundes erlangen. Freilich sollte man mit einer solchen Senderfusion nicht glauben machen, dass kurzfristig wesentliche Spareffekte erreicht werden können, im Gegenteil: Wenn die Gebührenbemessung ab 2005 grundsätzlich verändert werden soll, wäre die dann bestehende Zweiländeranstalt zunächst durchaus auf eine Finanzausgleichsregelung im ARD-Verband angewiesen. Die Fusionsabsicht wird im Übrigen von der ARD begrüßt. ${ }^{15}$

Eine Aufgabenkritik ergibt: Der Programmauftrag dieser Anstalt ist und bleibt die umfassende Berichterstattung in Hörfunk und Fernsehen aus Berlin und Brandenburg. Sollten aber nicht die polnischen Nachbarn mit einbezogen werden? Im Südwesten der Bundesrepublik ist der Umgang mit den französischen Nachbarn selbstverständlich geworden, liegt da nicht analog eine Zukunftsaufgabe für die Region Berlin und Brandenburg im Zuge der EU-Osterweiterung? Wie groß oder klein soll der direkte parteipolitische Einfluss, etwa in den Gremien, werden? Auch hier gibt es unterschiedliche Erfahrungen in beiden Bundesländern. ${ }^{16}$ Letzte Wahlergebnisse deuten darauf hin, dass der Wähler wählerischer geworden ist, so in Hamburg und Berlin. Wäre es nicht ratsam, die Amtszeit von Intendant, Rundfunkrat und Verwaltungsrat vom Wahlrhythmus der Politik „abzukoppeln“, d. h. durch eine längere Amtszeit dieser Organe für Kontinuität und Stabilität in der Rundfunklandschaft zu sorgen, zumal selbst Landesregierungen mit Doppelhaushalten bei wichtigen Strukturentscheidungen praktisch so arbeiten? (Ein Haushaltsgrundsatz ist ja die Jährlichkeit.) Dass unterschiedliche Amtszeiten zur Gewaltenteilung beitragen und damit Machtmissbrauch vermeiden helfen, ist bekannt. Auch ist die Beteiligung der Personalvertreter mit beschließender Stimme im für den Haushalt zuständigen Verwaltungsrat umstritten: Wird die betriebliche Mitbestimmung durch die Zustimmung zu Tarifverträgen und Intendantenverträgen durchgesetzt oder wird sie durch ein geborenes Stimmrecht im Verwaltungsrat sogar verdoppelt? Werden sich alle Teile des Landes, die Metropole, der engere Verflechtungsraum und die äußeren Regionen im Programm der gemeinsamen Landesrundfunkanstalt wiederfinden?

So stellt sich die beabsichtigte Senderfusion als eine realisierbare politische Option dar. Die Region Berlin und Brandenburg kann davon profitieren, wenn nicht die Fehler der Länderfusionsabsicht von 1995 wiederholt werden. Man muss damit rechnen, dass die Senderfusion von anderen Institutionen und von den Bürgern durchaus beobachtet wird und dass daraus Erfahrungen gewonnen und Folgerungen für andere Fusionen abgeleitet werden, um Fusionsfähigkeit und Fusionsbereitschaft zu beurteilen. In diesem Sinne ist das Vorhaben beispielgebend. Dass der Sitz einer fusionierten Anstalt die 
Brandenburger Landeshauptstadt Potsdam sein wird, liegt wohl ohne nähere Begründung auf der Hand. ${ }^{17}$ Ob damit die Sorgen der Bürger hinsichtlich der Verschuldung der Landeshaushalte ausgeräumt werden können, die eine später beabsichtigte Länderfusion begleiten, muss nach den Berliner Ereignissen des Jahres 2001 dagegen weiter offen bleiben.

\section{Fazit}

Für die Schaffung stärkerer Strukturen, etwa eines fusionierten Bundeslandes Berlin-Brandenburg, muss von den konkreten Gegebenheiten ausgegangen werden. Eine deutliche Bevölkerungsmehrheit in der Metropole würde in jeder Konstruktion eines Planungsverbandes die Majorisierung der Anteile des Landes Brandenburg nach sich ziehen. Demokratietheoretisch würde ein Planungsverband die Berliner Mehrheit fixieren, womit das Anliegen einer gemeinsamen Landesplanung konterkariert würde. - Daher wurde eine Gemeinsame Landesplanungsabteilung beider Bundesländer gebildet, die ein gestuftes Verfahren zur Dissens-Aufklärung kennt mit dem Ziel, ein länderübergreifendes Einvernehmen zu erzielen. Dies ist institutionell verankert worden und in Deutschland einmalig. Obwohl die Gemeinsame Landesplanung „der Diener zweier Herren“ ist, erweist sich ihre Arbeit als zweckdienlich, erfolgreich und womöglich beispielgebend.

\section{Anmerkungen}

1 Die Landesrundfunkanstalt des Saarlandes ist eine „kleine Anstalt", das Saarland erst 1957 zur Bundesrepublik gekommen.

2 Zeitpunkt des Inkrafttretens des Zweckverbandsgesetzes von 1911.

3 Der Abstand von 4 Wochen zwischen Regierungserklärung und Aussprache dazu ist wohl ohne Beispiel.

4 Mit der Einsetzung des ersten (und einzigen) Unterausschusses konnte der Vorsitz an einen Vertreter der stärksten Partei, also der Regierungspartei gegeben werden.

5 Ein entsprechender Antrag der PDS-Opposition im Landtag Brandenburg wurde abgelehnt.

6 Vertrag über die Aufgaben und Trägerschaft sowie Grundlagen und Verfahren der Gemeinsamen Landesplanung zwischen den Ländern Berlin und Brandenburg (Landesplanungsvertrag); Berlin GVBI. 1995 S. 407, Brandenburg: GVBI.I 1995, S. 210.

7 Landesentwicklungsplan Brandenburg Zentralörtliche Gliederung (LEP I) Brandenburg: GVBI.II 1995, S. 474.

8 Gemeinsames Landesentwicklungsprogramm der Länder Berlin und Brandenburg (LEPro) Berlin: GVBI. 1997, S. 657; Brandenburg GVBI. I 1998, S. 14.

9 Gemeinsamer Landesentwicklungsplan für den engeren Verflechtungsraum Brandenburg-Berlin (LEP eV) Berlin: GVBI. 1998, S. 38, Brandenburg: GVBI.II 1998, S. 186.

10 Gemeinsamer Landesentwicklungsplan Standortsicherung Flughafen (LEP SF) Berlin: GVBI. 1999, S. 121, Brandenburg: GVBI.II 1999, S. 262.
11 Gesetz zur Regionalplanung und zur Braunkohlen- und Sanierungsplanung (RegBkPIG) vom 15. März 2001 (GVBl. S. 170).

12 Der Begriff des Ständigen Vertreters wurde bereits zwischen der Bundesrepublik Deutschland und der DDR verwendet.

13 Man rechnete so mit 600000 Menschen.

14 Sollte sich die Olympia-Bewerbung einer Metropole erfolgreich gestalten, wäre der Fortgang der Regionalplanung besonders zu verfolgen.

15 So der ARD-Vorsitzende Pleitgen auf der Jahreshauptversammlung der ARD in Potsdam im November 2001.

16 Eingangs wurde auf den unterschiedlichen politischen Organisationsgrad verwiesen.

17 So der Brandenburger SPD-Landesvorsitzende Platzeck am 08.01.2001 im ORB.

\section{Autor}

\section{Dr. Dr. Markus Vette}

Technische Fachhochschule Wildau

Fachbereich Wirtschaft, Verwaltung und Recht

Tel. (0 33 75) 508-972

E-Mail:markus@vette-net.de

\section{Hinweis:}

Der vorstehende Artikel wurde im März 2002 verfasst und gibt daher nicht den aktuellen Entwicklungsstand zum Erscheinungstermin dieser Wissenschaftlichen Beiträge wieder. 